\title{
Identification of SUMO activating enzyme 1 inhibitors utilizing virtual screening approach
}

\author{
Ashutosh Kumar ${ }^{1 *}$, Akihiro Ito², Mikako Hirohama², Minoru Yoshida², Kam YJ Zhang ${ }^{1}$ \\ From 9th German Conference on Chemoinformatics \\ Fulda, Germany. 10-12 November 2013
}

Sumoylation is a post-translational modification affecting diverse cellular processes including DNA replication and repair, chromosome packing and dynamics, genome integrity, nuclear transport, signal transduction and cell proliferation [1]. Sumoylation involves the covalent attachment of a small ubiquitin like modifier (SUMO) protein to $\varepsilon$-amino group of lysine residues in specific target proteins via a sequential action of an activating enzyme E1 (SUMO E1), a conjugating enzyme E2 and a ligase E3. Among the sumoylation proteins, SUMO E1 is responsible for the activation of SUMO in the first step of the sumoylation cascade [2]. SUMO E1 is linked to many human diseases including cancer and thus making it a potential therapeutic target [3]. However, only a few inhibitors were reported up to now that includes three natural products, semi-synthetic protein inhibitors and one AMP mimic [4-6]. Here in this research, the combination of structure based virtual screening and in vitro sumoylation assay was used to identify potential small molecule inhibitors of SUMO E1 that could be used in chemical biology and therapeutic studies. Our virtual screening protocol involves the fast docking of a small molecule library to rigid protein followed by redocking of top hits using a method that incorporates both ligand and protein flexibility. Subsequently, the top ranking compounds were prioritized using molecular dynamics simulation based binding free energy calculation. The result of biological assay and subsequent similarity search resulted in the identification of two classes of small molecules that shared biaryl urea scaffold. Both of these chemical classes displayed moderate inhibitory potency against SUMO E1. The most potent compound of each class inhibited the in vitro sumoylation with an $\mathrm{IC}_{50}$ of 11.1 and $13.4 \mu \mathrm{M}$. These compounds inhibit sumoylation by blocking the formation of SUMO-E1 thioester intermediate. Our study presents starting points for the development of novel therapeutic agents against various diseases targeting SUMO E1.

\section{Authors' details}

${ }^{1}$ Zhang Initiative Research Unit, Institute laboratories, RIKEN, 2-1 Hirosawa, Wako, Saitama 351-0198, Japan. ${ }^{2}$ Chemical Genetics Laboratory/Chemical Genomics Research Group, RIKEN, 2-1 Hirosawa, Wako, Saitama 351-0198, Japan.

Published: 11 March 2014

\section{References}

1. Geiss-Friedlander R, Melchior F: Concepts in sumoylation: a decade on. Nat Rev Mol Cell Biol 2007, 8:947-956.

2. Lois LM, Lima CD: Structures of the SUMO E1 provide mechanistic insights into SUMO activation and E2 recruitment to E1. EMBO J 2005, 24:439-451.

3. Kessler JD, Kahle KT, et al: A SUMOylation-Dependent Transcriptional Subprogram Is Required for Myc-Driven Tumorigenesis. Science 2012, 335:348-353.

4. Fukuda I, Ito A, et al: Ginkgolic acid inhibits protein SUMOylation by blocking formation of the E1-SUMO intermediate. Chem Biol 2009, 16:133-140.

5. Lu X, Olsen SK, et al: Designed semisynthetic protein inhibitors of Ub/Ubl E1 activating enzymes. J Am Chem Soc 2010, 132:1748-1749.

6. Soucy TA, et al: An inhibitor of NEDD8-activating enzyme as a new approach to treat cancer. Nature 2009, 458:732-736.

\section{doi:10.1186/1758-2946-6-S1-P37}

Cite this article as: Kumar et al:: Identification of SUMO activating enzyme 1 inhibitors utilizing virtual screening approach. Journal of Cheminformatics 2014 6(Suppl 1):P37. 